\title{
Nível de testosterona total em mulheres pós-menopausa com olho seco
}

\author{
Total testosterone level in postmenopausal women with dry eye
}

\author{
Maria Cecília Barros Duarte ${ }^{1}$ \\ Natércia Trindade Pinto ${ }^{2}$ \\ Hamilton Moreira ${ }^{3}$ \\ Ana Tereza Ramos Moreira ${ }^{4}$ \\ Daniel Wasilewski ${ }^{5}$
}

\section{RESUMO}

Objetivo: Comparar o nível sérico de testosterona total entre mulheres pósmenopausa divididas em 3 grupos: controle, olho seco leve a moderado e olho seco intenso. Métodos: No ambulatório de climatério foram selecionadas 29 mulheres pós-menopausa que não faziam reposição hormonal há pelo menos 8 semanas. Critérios de exclusão: alterações palpebrais mecânicas, pterígio, obstrução de vias lacrimais, inflamação intra-ocular e uso de lente de contato. As mulheres selecionadas foram submetidas à dosagem de testosterona total, aplicação do questionário OSDI (Ocular Surface Disease Index) e exame oftalmológico para detecção de olho seco. As mulheres foram divididas em 3 grupos conforme o resultado do escore OSDI e do exame oftalmológico. Resultados: Cinco pacientes foram classificadas como ausência de olho seco, 15 com olho seco leve a moderado e 4 com olho seco intenso. Não houve diferença estatisticamente significativa entre a média dos valores das idades $(\mathrm{p}=0,3915)$; nível de instrução $(\mathrm{p}=0,9333)$; doenças associadas ( $p=0,2551)$; tipo de medicação utilizada $(p=0,2844)$ e nível sérico de testosterona total entre os grupos $(\mathrm{p}=0,1275)$. Conclusão: Não encontramos diferença estatisticamente significativa entre o nível de testosterona total entre mulheres pós-menopausa com olho seco. Novos estudos clínicos com maior amostra são necessários para melhor esclarecer a relação dos níveis séricos dos androgênios nos portadores de olho seco.

Descritores: Síndromes do olho seco; Testosterona; Hormônios; Pós-menopausa; Questionários

\section{INTRODUÇÃO}

A ceratoconjuntivite seca, ou síndrome do olho seco define-se como uma desordem do filme lacrimal por diminuição da produção ou por evaporação excessiva de lágrima que causa dano à superfície ocular e está associada a sintomas de desconforto ocular ${ }^{(1)}$.

Olho seco é um dos problemas mais freqüentes na prática oftalmológica em todo mundo. Na Alemanha, 1 em cada 4 pacientes que consultam o oftalmologista queixam-se de sintomas de olho $\operatorname{seco}^{(2)}$. Estudos epidemiológicos que investigam a prevalência do olho seco são raros, apesar disso, alguns trabalhos publicados indicam que aproximadamente $15 \%$ da população acima de 45 anos têm sintomas de olho $\operatorname{seco}^{(3)}$. As consequiências econômicas incluem utilização de recursos de assistência à saúde como visitas médicas, medicações e procedimentos cirúrgicos, além de diminuição da produtividade em decorrência dos sintomas irritativos ${ }^{(4)}$.

O olho seco é uma condição complexa com múltiplos fatores envolvidos. Na última década, pesquisas em animais e humanos evidenciaram o papel da inflamação da superfície ocular e do desequilíbrio dos hormônios 
sexuais (estrógeno/andrógeno) na patogênese do olho seco. Esses estudos sugerem que a deficiência de andrógenos gera um ambiente pro-inflamatório na superfície ocular e que níveis específicos de andrógenos são necessários para manter as funções secretórias e anti-inflamatórias de todo o sistema de superfície ocular, incluindo a glândula lacrimal e meibomiana ${ }^{(5)}$.

Este estudo teve como objetivo comparar o nível sérico de testosterona total entre mulheres pós-menopausa divididas em 3 grupos: controle, olho seco leve a moderado e olho seco intenso.

\section{MÉTODOS}

Esse é um estudo descritivo transversal realizado no ambulatório de oftalmologia do Hospital de Clínicas da Universidade Federal do Paraná (UFPR) em parceria com o serviço de ginecologia desse mesmo hospital. No ambulatório de climatério foram selecionadas mulheres pós-menopausa (definido como: 1 ano após a cessação dos ciclos menstruais) que não estivessem em terapia de reposição hormonal há pelo menos 8 semanas. Foram excluídas do estudo as mulheres com alterações mecânicas palpebrais (entrópio, ectrópio, lagoftalmo, retração palpebral); pterígio; obstrução de vias lacrimais, processos inflamatórios intra-oculares e usuárias de lente de contato. Foram estudadas 29 mulheres no período de 22 de novembro de 2004 a 24 de janeiro de 2005. Esse estudo foi aprovado pelo Comitê de Ética em Pesquisa do Hospital de Clínicas da UFPR (938.168/2004-10). Todas as participantes assinaram um termo de consentimento informado.

\section{Dosagem de testosterona total}

As mulheres selecionadas para o estudo eram orientadas a fazer uma coleta de sangue no laboratório do Hospital de Clínicas durante o período da manhã, na semana do exame oftalmológico. A dosagem da testosterona total foi realizada através de enzimaimunoensaio quimioluminescente competitivo de fase sólida. Das 29 pacientes, 2 não realizaram a coleta de sangue, sendo excluídas do estudo.

\section{Exame oftalmológico/detecção de olho seco}

No ambulatório de oftalmologia, após anamnese com atenção especial às doenças associadas e medicações de uso contínuo, o questionário do OSDI (Ocular Surface Disease Index) foi aplicado, de acordo com as orientações da empresa que o elaborou, tendo os últimos 7 dias como referência. Nessa oportunidade foram realizados ainda: medida da acuidade visual; refração; fundoscopia direta; biomicroscopia do segmento anterior; teste de quebra do filme lacrimal, teste de Schirmer e coloração com rosa bengala. Todos os exames, incluindo a anamnese e a aplicação do questionário foram realizados pelo mesmo examinador. $\mathrm{Na}$ anamnese as pacientes relatavam todos os medicamento de uso contínuo e esses eram agrupados em: 0) ausência de medicação; 1) medicamento associado com olho seco segundo a literatura; 2- medicamento em que desconhece-se relação com olho seco. Ne- nhuma das pacientes fazia uso de medicação tópica ocular. O primeiro teste realizado foi o tempo de quebra do filme lacrimal $(\text { BUT })^{(6)}$. Uma gota de fluoresceína $1 \%\left(\right.$ Ophthalmos $\left.^{\circledR}\right)$ era instilada no fórnice inferior de ambos os olhos; as pacientes piscavam 2 a 3 vezes e então solicitava-se que permanecessem com os olhos abertos até que o primeiro ponto de quebra do filme lacrimal fosse identificado na córnea com luz azul de cobalto na lâmpada de fenda. $\mathrm{O}$ tempo entre o último piscar e o aparecimento do primeiro ponto de quebra do filme lacrimal era registrado. Posteriormente as glândulas meibomianas eram examinadas à lâmpada de fenda. A disfunção da glândula meibomiana (DGM) foi graduada em: grau 0 - sem obstrução; grau 1 - menos de um terço das glândulas obstruídas; grau 2 mais de um terço das glândulas obstruídas com secreção espessa; grau 3 - mais da metade das glândulas obstruídas sem secreção à expressão. Após a avaliação das glândulas meibomianas, uma gota de anestésico (Allergan ${ }^{\circledR}$ ) era instilada no fórnice inferior de ambos os olhos das pacientes, com um cotonete enxugava-se o fundo de saco conjuntival e após 3 minutos o papel filtro milimetrado $\left(\mathrm{Ophthalmos}{ }^{\circledR}\right)$ para realização do teste de Schirmer era colocado entre o terço médio e o terço externo da pálpebra inferior. Após 5 minutos cronometrados o papel era retirado e registrava-se a leitura ${ }^{(6)}$. Por fim seguiu-se a coloração com rosa bengala através de tiras de rosa bengala $\left(\mathrm{Ophthalmos}^{\circledR}\right)$ umedecidas com água destilada e instiladas no fórnice inferior de ambos os olhos. Observou-se a localização e a intensidade da impregnação da conjuntiva nasal, córnea e conjuntiva temporal segundo o método de van Bijsterveld ${ }^{(7)}$. A intensidade de coloração dessas três áreas foi quantificada em uma escala numérica para cada região, variando de 0 a 3 cruzes, na qual 0 significava ausência de coloração; 1) coloração puntiforme; 2) pontos confluentes e 3) placas extensas de coloração. Realizou-se o somatório da pontuação de cada região.

Durante a anamnese duas pacientes foram excluídas, a primeira por ter sido submetida a ceratotomia radial há 10 anos, e a segunda por estar em uso de isoflavona, informações essas não obtidas na seleção das candidatas no ambulatório de climatério. À lâmpada de fenda uma terceira paciente foi excluída do estudo por apresentar um pterígio recidivado não identificado anteriormente.

\section{Formação dos grupos}

As pacientes foram divididas em 3 grupos: ausência de olho seco (grupo controle) $(n=5)$, olho seco leve a moderado $(n=15)$ e olho seco intenso $(n=4)$. Integraram o grupo controle todas as mulheres em pós-menopausa com escore de OSDI $<10$; BUT $\geq 10$ segundos em ambos os olhos; ausência de meibomite ou meibomite grau 1 em ambos os olhos; coloração com rosa bengala $<3$ e Schirmer $>5 \mathrm{~mm}$ em ambos os olhos. Sinais positivos de olho seco leve a moderado foram definidos como pelo menos 1 dos seguintes itens em um dos olhos: OSDI $\geq 10$; BUT $<10$; rosa bengala $>3$; Schirmer $\leq 5 \mathrm{~mm}$. No grupo de olho seco intenso foram agrupadas as mulheres pós-menopausa que apresentavam pelo menos três resultados positivos den- 
tre os seguintes itens: OSDI $>30$; BUT $<5$ em um dos olhos; rosa bengala $\geq 6$ em um dos olhos; Schirmer $\leq 5 \mathrm{~mm}$ em um dos olhos (Tabela 1).

\section{Análise estatística}

Por não ser verificada normalidade nos dados coletados, foi utilizado o teste não paramétrico de Kruskal-Wallis. Considerou-se estatisticamente significativo os valores de $\mathrm{p}<0,05$ para um intervalo de confiança de $95 \%$.

\section{RESULTADOS}

A média de idade no grupo controle foi de 54,40 $\pm 2,70$ anos (variando entre 51,05 e 57,75 anos); no grupo com olho seco leve a moderado a média foi de 54,73 $\pm 7,48$ anos (variando entre

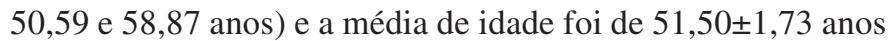
(variando entre 48,74 e 54,26 anos) no grupo com olho seco intenso $(\mathrm{p}=0,3915)$. O tempo médio de pós-menopausa foi de $7 \pm 2,92$ (variando entre 3,38 e 10,62 anos) no grupo controle; $10,30 \pm 9,17$ (variando entre 5,22 e 15,38 anos) no grupo com olho seco leve a moderado e $12 \pm 6,48$ (variando entre 1,69 e 22,31 anos) no grupo com olho seco severo ( $\mathrm{p}=0,4238)$.

Quanto ao nível de instrução das mulheres, não houve diferença estatisticamente significativa entre os grupos $(p=0,9333)$. A grande maioria das mulheres (79\%) possuía $1^{\circ}$ grau incompleto.

Não houve diferença estatisticamente significativa no número de doenças associadas entre os grupos ( $p=0,2551)$. A condição mais freqüentemente encontrada foi a hipertensão arterial sistêmica, tanto nos grupos em separado quanto em conjunto.

Das 24 participantes, apenas 4 não faziam uso contínuo de medicação sistêmica. No grupo controle, a média do número

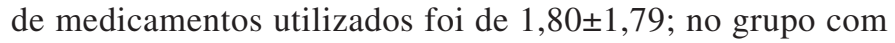
olho seco leve a moderado a média foi de 2,73 $\pm 1,91$ e no grupo

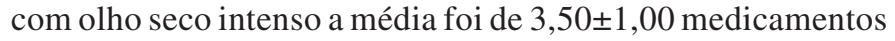
$(\mathrm{p}=0,3145)$. Analisando quanto ao grupo da medicação $(0 ; 1$ ou 2) conforme descrição acima, não houve diferença estatisticamente significativa entre os grupos $(\mathrm{p}=0,2844)$, sendo que os medicamentos associados ao olho seco foram os mais freqüentemente identificados tanto nos grupos em separado quanto em conjunto.

A média dos valores séricos de testosterona total no grupo controle foi de $34,36 \pm 9,78 \mathrm{ng} / \mathrm{dL}$; no grupo com olho seco leve a moderado a média foi de $29,17 \pm 16,75 \mathrm{ng} / \mathrm{dL}$ e no grupo com olho seco intenso a média foi de $22,63 \pm 5,25 \mathrm{ng} / \mathrm{dL}(\mathrm{p}=0,1275)$ (Figura 1).

\section{DISCUSSÃO}

Esse estudo comparou os níveis de testosterona total em mulheres pós-menopausa divididas em 3 grupos: sem olho seco (grupo controle), olho seco leve a moderado e olho seco intenso. Observamos que a média dos níveis de testosterona total foi maior no grupo controle, menor no grupo com olho leve a moderado e menor ainda no grupo com olho seco intenso. No entanto, não houve diferença estatisticamente significativa entre os níveis de testosterona total entre os grupos $(\mathrm{p}=0,1275)$.

$\mathrm{O}$ presente estudo apresenta algumas limitações. Em primeiro lugar, o tamanho da amostra é pequeno. Durante os 2 meses de seleção apenas 29 mulheres preencheram os critérios de inclusão, isso deve-se ao fato de que o ambulatório de climatério é freqüentado principalmente por mulheres em terapia de reposição hormonal, critério de exclusão desse estudo. Em segundo lugar, os grupos foram formados segundo critérios definidos pelos autores, os quais foram baseados no questionário do OSDI e nos testes clássicos de detecção de olho seco. Ressaltamos aqui, a dificuldade encontrada na aplicação do questionário do OSDI na amostra estudada. Tal fato é explicado pelo baixo nível de instrução das pacientes (79\% das mulheres não completaram o primeiro grau), combi-

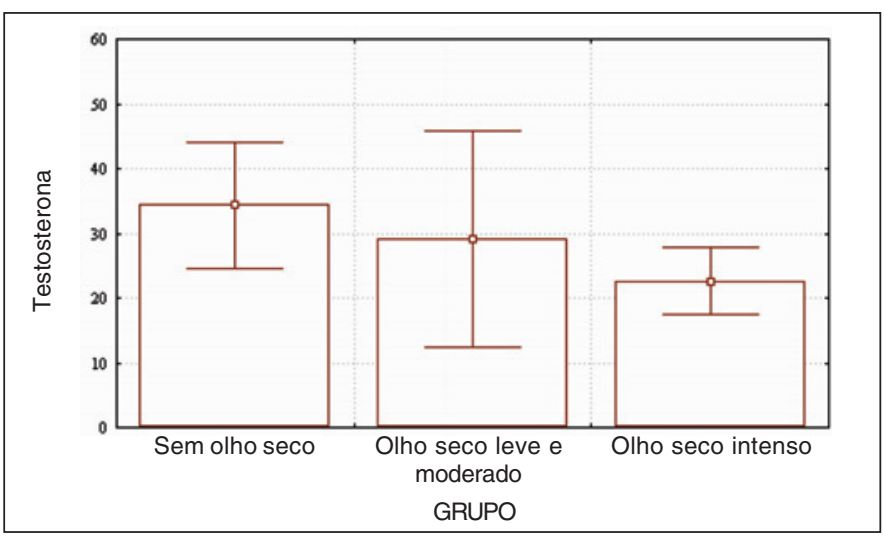

Figura 1 - Níveis de testosterona total nas mulheres pós-menopausa analisadas

\begin{tabular}{|c|c|c|c|c|c|c|c|c|}
\hline & \multicolumn{6}{|c|}{ Grupo } & \multirow{2}{*}{\multicolumn{2}{|c|}{$p^{*}$}} \\
\hline & \multicolumn{2}{|c|}{ Sem olho seco $(n=5)$} & \multicolumn{2}{|c|}{ Olho seco leve a moderado $(n=15)$} & \multicolumn{2}{|c|}{ Olho seco intenso $(n=4)$} & & \\
\hline & OD & OE & OD & OE & OD & OE & OD & OE \\
\hline BUT & $11,20 \pm 2,17$ & $12,40 \pm 5,03$ & $7,33 \pm 3,74$ & $7,00 \pm 3,70$ & $4,25 \pm 2,06$ & $5,75 \pm 2,99$ & $0,0158^{\dagger}$ & 0,0705 \\
\hline Schirmer & $13,50 \pm 3,42$ & $13,75 \pm 4,35$ & $13,13 \pm 10,19$ & $14,13 \pm 9,12$ & $4,00 \pm 1,41$ & $6,25 \pm 2,87$ & $0,02866^{\dagger}$ & 0,0559 \\
\hline Rosa Bengala & $2,75 \pm 2,06$ & $0,50 \pm 0,58$ & $2,20 \pm 2,18$ & $1,33 \pm 0,72$ & $4,50 \pm 2,65$ & $3,75 \pm 2,22$ & 0,2169 & $0,0132 \dagger$ \\
\hline
\end{tabular}


nado à falta de recursos financeiros, condições que caracterizam a população atendida em hospitais públicos em países em desenvolvimento. O OSDI é um questionário específico para olho seco e pergunta aos pacientes a freqüência desses sintomas e seu impacto na função visual ${ }^{(8)}$. No entanto as questões que investigam a relevância dos sintomas na função visual são as mais difíceis de serem respondidas, já que o uso de computadores; o hábito da leitura e a condução de veículos não fazem parte da rotina dessas mulheres. Seria útil que um questionário diferenciado para diagnóstico de olho seco em países em desenvolvimento fosse desenvolvido, trazendo perguntas mais condizentes com a realidade dessas populações. Além disso, os resultados dos testes clínicos atuais para detecção de olho seco correlacionam-se pobremente com os sintomas relatados pelos pacientes ${ }^{(3,9-11)}$, tornando confuso o diagnóstico e a graduação dessa doença.

Outra limitação do estudo deve-se ao método utilizado para a dosagem da testosterona total: enzimaimunoensaio quimioluminescente competitivo de fase sólida. Esse método não detecta valores de testosterona total menores que $20 \mathrm{ng} / \mathrm{dL}$ ou $0,2 \mathrm{ng} / \mathrm{mL}$, sendo que para todas as mulheres com resultados menores que $20 \mathrm{ng} / \mathrm{mL}(\mathrm{n}=12)$ foi assumido o valor $20 \mathrm{ng} / \mathrm{mL}$ para os cálculos de estatística. Por fim, das 29 mulheres selecionadas, apenas 4 não faziam uso contínuo de medicação sistêmica, a qual tem sido considerada um fator predisponente para olho $\operatorname{seco}^{(12-15)}$. Os grupos de drogas apontados na literatura como responsáveis por essa associação são principalmente os antidepressivos, diuréticos, anti-colinérgicos e anti-histamínicos. Identificamos mulheres em uso dessas drogas nos 3 grupos, não havendo, no entanto, diferença significativa quanto a essa variável entre os grupos. Além disso, a análise estatística também não mostrou diferença entre os grupos quanto ao número de drogas utilizadas pelas pacientes.

Desconhecemos outros estudos específicos que tenham avaliado o nível sérico de testosterona total nas mulheres pósmenopausa. Outros trabalhos relataram níveis diminuídos de testosterona em mulheres com olho seco ${ }^{(16)}$. Por outro lado, a dosagem de testosterona total não representa de forma confiável o "pool" total de andrógenos nos humanos, sendo mais válido os níveis séricos dos metabólitos conjugados da didrotestosterona (DHT) ${ }^{(17)}$. Em nosso estudo utilizamos a dosagem da testosterona total por ser a única forma de avaliação dos andrógenos no laboratório do nosso hospital. A dosagem de metabólitos da DHT é cara e não está disponível na prática clínica.

Andrógenos modulam a anatomia, fisiologia, e o sistema imune da glândula lacrimal em ratos, coelhos, hamsters e humanos $^{(18)}$. Além da pós-menopausa, processo de envelhecimento, síndrome de Sjögren, síndrome de insensibilidade androgênica, uso de drogas antiandrogênicas ${ }^{(17,19)}$, lactação, gravidez ${ }^{(5)} \mathrm{e}$ várias outras condições cursam com níveis diminuídos de andrógenos. Nessa situação, a imunohomeostase da superfície ocular é afetada, levando à inflamação e a um estado de quiescência da glândula lacrimal. A importância de níveis de andrógenos adequados, bem como o equilíbrio andrógeno/ estrógeno torna-se cada vez mais evidente para a manutenção da saúde e integridade da superfície ocular e do filme lacrimal ${ }^{(5)}$.

Nesse estudo realizado com uma amostra das pacientes do serviço de ginecologia do HC-UFPR, apesar de ser perceptível a diferença entre os níveis de testosterona total entre os grupos estudados, essa diferença não foi estatisticamente significativa. Novos estudos clínicos são necessários para melhor esclarecer a relação entre os níveis séricos dos hormônios sexuais e a síndrome de olho seco.

\section{ABSTRACT}

Purpose: The purpose of this study was to compare total testosterone blood level among three groups of postmenopausal women: control, mild to moderate dry eye and severe dry eye. Methods: Twenty-nine postmenopausal women were selected. The exclusion criteria were: hormone replacement therapy in the last 8 weeks, mechanical palpebral abnormalities, pterygium, lacrimal obstructions, intraocular inflammation or contact lens use. A blood sample was collected for total testosterone level determination, and the patients were submitted to an ophthalmologic examination (emphasizing on dry eye detection) and answered the OSDI (Ocular Surface Disease Index) questionnaire. Five patients were excluded. Postmenopausal women were divided into three groups according to OSDI score and the ophthalmic examination. Results: Five patients were classified in the no dry eye group (control), fifteen in the mild to moderate dry eye group and four in the severe dry eye group. There were no statistically significant differences regarding mean age $(p=0.3915)$; instruction level ( $p=0.9333)$; number of comorbidities $(p=0.2551)$; medication taken $(p=0.2844)$ and total testosterone level among those groups ( $p=0.1275$ ). Conclusion: Further research with a greater bigger sample is necessary to establish the relation of androgen levels in dry eye patients.

Keywords: Dry eye syndromes; Testosterone; Hormones; Postmenopause; Questionaires

\section{REFERÊNCIAS}

1. Lemp MA. Report of the National Eye Institute/Industry workshop on Clinical Trials in Dry Eyes. CLAO J. 1995;21(4):221-32.

2. Brewitt H, Sistani F. Dry eye disease: the scale of the problem. Surv Ophthalmol. 2001;45. Suppl 2: S199-202.

3. Moss SE, Klein R, Klein BE. Prevalence of and risk factors for dry eye syndrome. Arch Ophthamol. 2000;118(9):1264-8.

4. Reddy P, Grad O, Rajagopalan K. The economic burden of dry eye: a conceptual framework and preliminary assessment. Cornea. 2004;23(8):751-61.

5. Smith RE. The tear film complex: pathogenesis and emerging therapies for dry eyes. Cornea. 2005;24(1):1-7.

6. Farris RL. Tear film evaluation. In: Krachmer JH, Mannis MJ, Holland EJ, editors. Cornea: fundamentals of cornea and external disease. St. Louis: Mosby; 1997.v.1. p.271-2.

7. Van Bijsterveld OP. Diagnostic tests in the Sicca syndrome. Arch Ophthalmol. 1969;82(1):10-4. 
8. Schiffman RM, Christianson MD, Jacobsen G, Hirsch JD, Reis BL. Reliability and validity of the Ocular Surface Disease Index. Arch Ophthamol. 2000; 118(5):615-21.

9. Nichols KK, Nichols JJ, Mitchell GL. The lack of association between signs and symptoms in patients with dry eye disease. Cornea. 2004;23(8):762-70.

10. Pflugfelder SC, Solomon A, Stern ME. The diagnosis and management of dry eye. Cornea. 2000;19(5):644-9. Review.

11. Bron AJ. Diagnosis of dry eye. Surv Ophthalmol. 2001;45. Suppl 2:S221-6. Review.

12. Bergmann MT, Newman BL, Johnson NC Jr. The effect of a diuretic (hydrochlorothiazide) on tear production in humans. Am J Ophthalmol. 1985; 99(4):473-5.

13. Norn M. The effects of drugs on tear flow. Trans Ophthalmol Soc U K. 1985; 104(Pt 4):410-4.

14. Schein OD, Hochberg MC, Munoz B, Tielsch JM, Bandeen-Roche K, Pro- vost T, et al. Dry eye and dry mouth in the elderly: a population-based assessment. Arch Intern Med. 1999;159(12):1359-63.

15. Moss SE, Klein R, Klein BE. Incidence of dry eye in an older population. Arch Ophthalmol. 2004;122(3):369-73.

16. Mamalis N, Harrison DY, Hiura G, et al. Dry eyes and testosterone deficiency in women [abstract]. In: Centennial Annual Meeting of the American Academy of Ophthalmology 1996, Chicago, IL; 1996. p.132.

17. Sullivan DA, Belanger A, Cermak JM, Berube R, Papas AS, Sullivan RM, et al. Are women with Sjogren's syndrome androgen-deficient. J Rheumatol. 2003;30(11):2413-9.

18. Baudouin C. The pathology of dry eye. Surv Ophthalmol. 2001;45. Suppl 2:S211-20. Review.

19. Krenzer KL, Dana MR, Ullman MD, Cermak JM, Tolls DB, Evans JE, Sullivan DA. Effect of androgen deficiency on the human meibomian gland and ocular surface. J Clin Endocrinol Metab. 2000;85(12):4874-82.

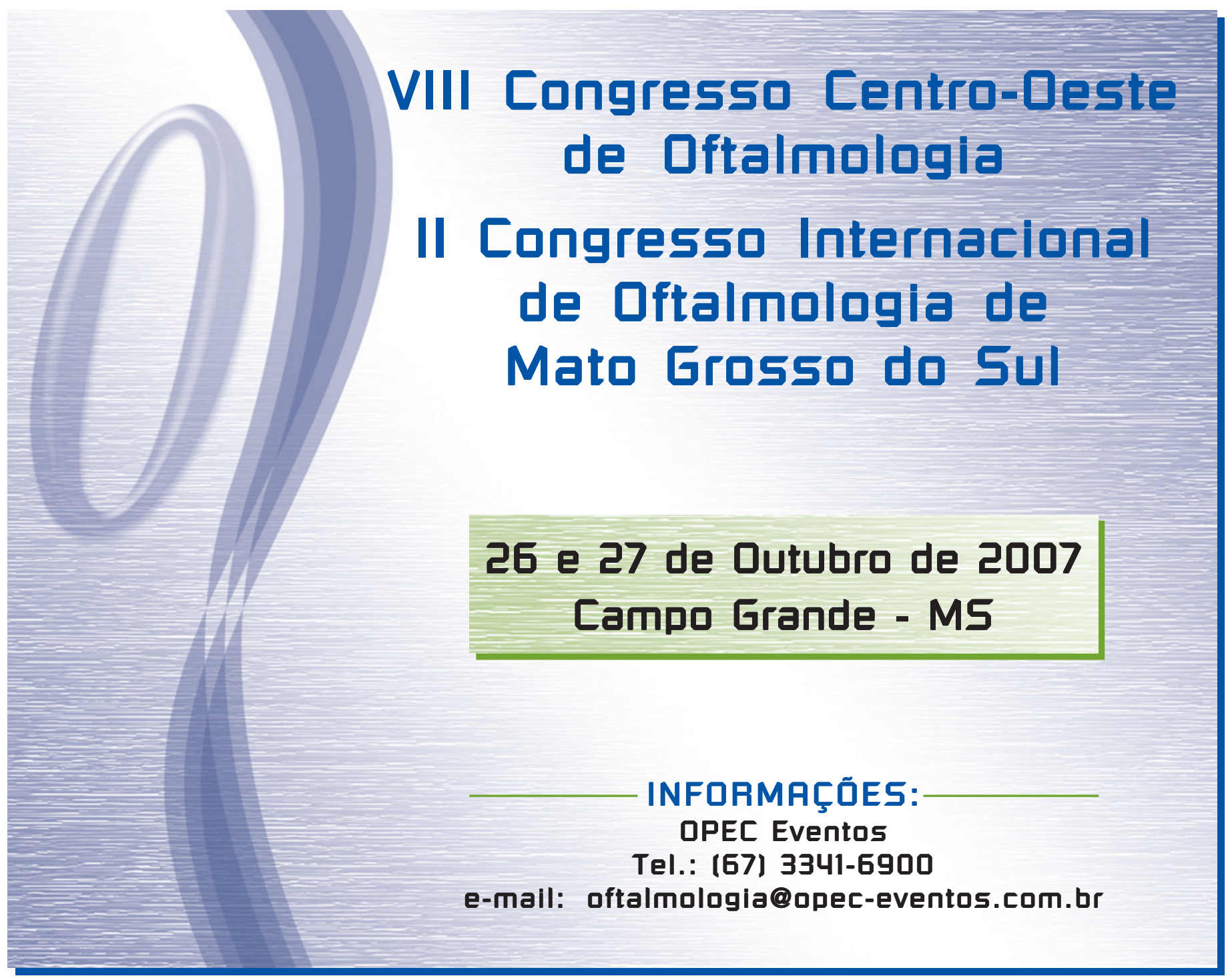

